\title{
Alternativas a la sujeción mecánica en un paciente con demencia vascular avanzada en tratamiento de hemodiálisis
}

\author{
Marta Del Vas, Elena Cahuancama, Carolina Palomar, Montserrat Cerro, Marta Quíntela, María del Transito \\ Suárez
}

Servicio de Nefrología. Hospital Clínic. Barcelona. España

Como citar este artículo: Del Vas M, Cahuancama E, Palomar C, Cerro M, Quíntela M, Suárez MT. Alternativas a la sujeción mecánica en un paciente con demencia vascular avanzada en tratamiento de hemodiálisis. Enferm Nefrol. 2020 Ene-Mar;23(1):94-97

\section{Resumen}

Descripción del caso: Varón de 90 años en tratamiento de hemodiálisis desde el año 2009 a través de Fistula arteriovenosa autológa en brazo izquierdo. En el 2018 comienza con deterioro cognitivo que finalmente diagnostican como demencia vascular avanzada. En las sesiones de hemodiálisis presenta desconexión del medio, agitación psicomotriz llegando en ocasiones a agresiones verbales e intentos de extracción de las agujas.

Descripción del plan de cuidados: Se realiza una valoración focalizada inicial para determinar la comorbilidad, mortalidad, el estado cognitivo y el grado de dependencia para las actividades de la vida, posteriormente aplicamos los diagnósticos de enfermería según la taxonomía NANDA con sus respectivos NIC y NOC.

Evaluación del plan: Entre el mes de diciembre del 2018 y enero de 2019, se valoró durante 24 sesiones el estado del paciente, para priorizar el uso de sujeción física o abordaje terapéutico alternativo, recurriendo a la musicoterapia, acompañamiento emocional, escucha activa y distracción audiovisual para disminuir la agitación psicomotriz. Las medidas alternativas a la sujeción física disminuyeron los episodios de agitación psicomotriz durante el tratamiento de hemodiálisis.

\section{Correspondencia:}

Marta Del Vas García

E-mail: martadelvas@hotmail.com
Conclusiones: El papel de enfermería es fundamental para evitar sujecciones físicas innecesarias. La única indicación para el uso de sujeción física se debería contemplar cuando existe el riesgo de interrupción del tratamiento, comprometiendo la seguridad del paciente. En el caso clínico presentado, las intervenciones alternativas a la sujeción física disminuyeron la agitación psicomotriz durante las sesiones de HD.

PALABRAS CLAVE: hemodiálisis; demencia vascular; restricción física; musicoterapia; atención de enfermería.

\section{Alternatives to mechanical restraint in a patient with advanced vascular dementia in hemodialysis treatment}

\section{Abstract}

Case description: A 90-year-old male on hemodialysis treatment since 2009 through autologous arteriovenous fistula in the left arm. In 2018, the patient presents cognitive deterioration symptoms, being finally diagnosed with advanced vascular dementia. In the hemodialysis sessions, he presents disconnection of the environment, psychomotor agitation, sometimes reaching verbal aggressions and attempts to extract the needles.

Description of the care plan: An initial focused assessment is carried out to determine comorbidity, 
mortality, cognitive status and the degree of dependence for activities of daily living, then nursing diagnoses according to the NANDA taxonomy, with their respective NIC and NOC, were applied.

Evaluation of the care plan: During the month of December 2018 and January 2019, the patient's condition was assessed for 24 sessions, to avoid the use of physical support or alternative therapeutic approach. Music therapy, emotional accompaniment, active listening and audio-visual distraction were used to reduce psychomotor agitation. Alternative measures to physical restraint decreased episodes of psychomotor agitation during hemodialysis treatment.

Conclusions: The nursing role is essential to avoid unnecessary physical restraint. The only indication for the use of physical restraint should be considered when there is a risk of interruption of treatment, compromising patient safety. In the case report presented, alternatives to physical restraint interventions decreased psychomotor agitation during hemodialysis sessions.

KEYWORDS: hemodialysis; vascular dementia; physical restraint; music therapy; nursing care.

\section{Introducción}

La edad de los pacientes en hemodiálisis (HD) ha aumentado considerablemente en los últimos años, influenciada por el aumento de la esperanza de vida en la población. La prevalencia de demencia en mayores de 65 años se sitúa en un $10 \%$ y aumenta conforme avanza la edad en la población. La frecuencia de la alteración cognitiva moderada-severa presenta mayor variabilidad cifrándose entre el 6-42\% ${ }^{1}$. Estos factores, junto con el elevado riesgo cardiovascular de las personas con enfermedad renal crónica (ERCA), ha aumentado la incidencia de trastornos cognitivos entre los pacientes con tratamiento de hemodiálisis².

Uno de los métodos aplicados a las personas con agitación psicomotriz es las sujeción física, definida como "cualquier dispositivo, material o equipo aplicado a una persona, unida a ella o cerca de su cuerpo, que no puede ser controlado o retirado con facilidad por ella misma y que deliberadamente evita o intenta evitar su libertad de movimiento y/o el natural acceso a su cuerpo" ${ }^{\prime \prime}$, siendo el recurso habitual para evitar daños provocados por el estado de agitación ${ }^{4}$, pero no siempre se consigue el efecto deseado, por el contrario, algunas veces la agitación aumenta, hecho que provoca mala tolerancia a la sesión de HD. Es por esta razón que el personal de enfermería se enfrenta a situaciones de alta complejidad que le exigen nuevos retos para poder garantizar la seguridad del paciente durante la sesión de diálisis, por ello es necesario buscar un abordaje terapéutico alternativo.

Presentamos un caso clínico sobre un paciente con demencia vascular avanzada en HD y las intervenciones alternativas a la sujeción física encaminadas a disminuir la agitación psicomotriz durante las sesiones de HD.

\section{Presentación del caso}

Varón de 90 años, con diagnóstico de enfermedad renal crónica de etiología no filiada desde el año 2009, que precisó tratamiento de HD a través de fístula arteriovenosa autóloga (FAVI) en extremidad superior izquierda. Soltero, sin hijos, vive solo y autónomo para las actividades de su vida diaria.

Antecedentes: Hipertensión arterial, diabetes mellitus tipo 2 con tratamiento ADOS, dislipemia, carcinoma renal derecho que requirió nefrectomía parcial derecha en el año 1992, cardiopatía isquémica con colocación de Stent en el año 2002. En el 2018 comienza con deterioro cognitivo que finalmente diagnostican como demencia vascular avanzada, precisando ayuda parcial para las actividades de la vida diaria e ingreso en centro socio sanitario por mayor compromiso funcional y cognitivo, sin disponibilidad familiar para su cuidado.

Durante las sesiones de HD presentaba desconexión del medio en tiempo y espacio, agitación psicomotriz llegando en ocasiones a agresiones verbales e intentos de retirada accidental de las agujas.

\section{Valoración enfermera focalizada}

Tras la nueva situación del paciente decidimos hacer una valoración inicial para determinar la comorbilidad, riesgo de mortalidad, el estado cognitivo y el grado de dependencia para las actividades de la vida diaria (Tabla 1).

\section{Descripción del plan de cuidados}

Para la fase de Diagnóstico utilizamos la Taxonomía NANDA con los objetivos enfermeros (NOC) propuestos para cada diagnóstico y las intervenciones enfermera (NIC) con las actividades a realizar (Tabla 2). 
Tabla 1. Resultados de la valoración focalizada con escalas e índices en diciembre de 2018.

\begin{tabular}{|l|c|l|}
\hline Test/Escala & Resultado & Interpretación del resultado \\
\hline Índice Charlson & 11 Puntos & Comorbilidad y mortalidad alta \\
\hline Test de Pfeiffer & 9 Errores & Deterioro cognitivo grave \\
\hline Índice Barthel & 10 Puntos & Dependencia total \\
\hline Escala de Lawton-Brody & 0 Puntos & Máxima dependencia \\
\hline
\end{tabular}

Tabla 2. Plan de cuidados con taxonomías NANDA/NIC/NOC aplicados al caso.

\begin{tabular}{|c|c|c|}
\hline NANDA & NOC & NIC \\
\hline - 00038 Riesgo de traumatismo & - 1913 Estado de seguridad: lesión física & $\begin{array}{l}\text { - } 6486 \text { Manejo ambiental: seguridad } \\
\text { - } 6580 \text { Sujeción física } \\
\text { - } 6610 \text { identificación de riesgos }\end{array}$ \\
\hline - 00129 Confusión crónica & - 908 Memoria & $\begin{array}{l}\text { - } 6460 \text { Manejo de la demencia } \\
\text { - } 1202 \text { Identidad } \\
\text { - } 6654 \text { Vigilancia: seguridad }\end{array}$ \\
\hline - 00146 Ansiedad & $\begin{array}{l}\text { - } 1402 \text { Control de la ansiedad } \\
\text { - } 1405 \text { Control de los impulsos }\end{array}$ & $\begin{array}{l}\text { - } 4920 \text { Escucha activa } \\
\text { - } 5270 \text { Apoyo emocional } \\
\text { - } 5820 \text { Disminución de la ansiedad } \\
\text { - } 5880 \text { Técnica de relajación }\end{array}$ \\
\hline
\end{tabular}

Tras detectar los problemas en la valoración y establecer los diagnósticos NANDA, se planificó y desarrolló un plan de cuidados durante los meses de Diciembre 2018 y Enero del 2019, en un total de 24 sesiones de HD, con previa autorización de la familia, a través del consentimiento informado.

Se realizaron diferentes actividades alternativas a la sujeción física encaminadas a controlar la ansiedad, ejercitar la memoria y la propia identidad del paciente, cuyo orden vario en función del estado cognitivo y condición clínica en cada una de las sesiones de HD.

\section{Actividades realizadas:}

- Contención verbal: Comunicación directa comprensiva y persuasiva, se utilizó tono suave y tranquilo.

- Musicoterapia: Escucha activa de sus propias interpretaciones (cantautor de Jotas) y música de su época a través audífonos conectados a una Tablet, distracción audiovisual a través del uso de sonajeros tipo maraca y campana.

- Estimulación multisensorial: Acompañamiento a pie de cama con contacto físico a través de las manos, uso de guante de látex con agua caliente para mantener sensación de contacto físico con otra persona.
- Medidas ambientales: Disminución de la intensidad de luz de la sala de hemodiálisis, uso permanente de las 4 barandillas de la cama, mantenimiento de la cama al más bajo nivel del suelo y uso de manopla en la mano de la extremidad contraria a la FAVI.

- Sujeción física: Contención de una o las dos extremidades superiores por periodos de tiempo que dependían del estado de agitación del paciente, la aplicación fallida de otras alternativas y de la disponibilidad y compromiso del personal de enfermería.

En el $71 \%$ de las sesiones se realizó abordaje alternativo (musicoterapia, distracción audiovisual y acompañamiento personalizado de enfermería), en un $25 \%$ se aplicó tiempos cortos de sujeción física combinada con periodos más largos de medidas alternativas y en un $4 \%$ requirió sujeción física durante toda la sesión de hemodiálisis por su agitación psicomotriz.

\section{Evaluación del plan de cuidados}

La musicoterapia con escucha de sus propias interpretaciones fue el abordaje alternativo que obtuvo mejores resultados al despertarle recuerdos y emociones que generaron cambios en su conducta, reduciendo su estado de 
ansiedad y agitación, presentando una mejor tolerancia a las sesiones de hemodiálisis.

Las medidas alternativas a la sujeción física disminuyeron los episodios de agitación psicomotriz durante el tratamiento.

El papel de enfermería fue fundamental para evitar sujeción física innecesaria, optando por un plan de seguimiento individualizado, contemplando como primera opción medidas que no alteraren la libertad y dignidad del paciente.

La única indicación para el uso de sujeción física es cuando existe riesgo de interrupción del tratamiento, comprometiendo la seguridad del paciente.

\section{Discusión}

La literatura indica que el uso de las contenciones físicas aplicadas en el ámbito de la sanidad, no solo no son necesarias, sino que tiene efectos negativos para la persona objeto de ellas ${ }^{3-8}$.

Las contenciones físicas deben pasar a ser un recuerdo del pasado, recurriendo a un abordaje terapéutico alternativo que no altere los principios éticos básicos: autonomía, beneficencia, no maleficencia y justicia ${ }^{7}$, los cuatro principios relacionados con la restricción de libertad que supone la utilización de la sujeción física.

Las enfermeras de cualquier entorno de atención deben ser consciente de la legislación vigente en su ámbito local, su sector de salud y la población a la que se atiende, para implantar el uso de buenas prácticas y apoyar los entornos libres de contenciones ${ }^{8}$; siendo el equipo de enfermería la piedra angular en la sala de hemodiálisis, dado que está presente en todo el procedimiento, manteniendo y facilitando la correcta individualización de los cuidados.

En el caso clínico presentado, las intervenciones alternativas a la sujeción física disminuyeron la agitación psicomotriz durante las sesiones de HD.
Recibido: 15-10-19

Revisado: 22-11-19

Modificado: 13-12-19

Aceptado: 15-12-19

\section{Bibliografía}

1. Gil Nieto D, Perera Díaz M, Sevane Fernández L. Estado cognitivo del paciente de edad avanzada en programa de hemodiálisis. Rev Soc Esp Enferm Nefrol 2010;13(1):30-5.

2. Sánchez Lamolda MA, Malagón Rodríguez MA, Alonso Hernández N. Incidencias de las demencias en hemodiálisis. Apoyo al cuidador principal. Enferm Nefrol 2013;16(1):31-5.

3. Fundación Cuidados Dignos. Cómo se eliminan las sujeciones. Guía para la eliminación de sujeciones físicas y químicas en centros de atención socio sanitaria y domicilios. Zaragoza: El Justicia de Aragón; 2011.

4. Estévez Ramos R, Basset Machado I, Guerrero Lara M, López Vázquez J, Leal García M. La sujeción del paciente con agitación psicomotriz. Enf Neurol (Mex) 2011;10(1):32-8.

5. Ashcraft L, Bloss M, Anthony WA. Best practices: The development and implementation of "no force first" as a best practice. Psychiatr Serv. 2012;63(5):415-7.

6. Corral Cano J. Contenciones físicas: reflexión sobre su vigencia. [Trabajo Fin de Grado] Santander: Universidad de Cantabria; 2014. [Consultado 05 mar 2019]. Disponible en: https://repositorio.unican.es/xmlui/ bitstream/handle/10902/5581/CorralCanoJ.pdf?sequence $=1$ \&isAllowed $=y$.

7. Burgueño A, Heras C. Centros libres de sujeciones físicas: un estándar de oro de calidad. Rev. Arg. de Gerontología y Geriatría; 31(3):77-82.

8. Asociación Profesional de Enfermeras de Ontario (RNA0). Guía de Buenas Prácticas Clínicas. Fomento de la seguridad: alternativas al uso de contenciones. Toronto (ON): Registered Nurses' Association of Ontario; 2012.

Este artículo se distribuye bajo una Licencia Creative Commons Atribución-NoComercial 4.0 Internacional. https://creativecommons.org/licenses/by-nc/4.0/ 